\title{
Molecular Level Differences in Ionic Solvation and Transport Behavior in Homopolymer and Block Copolymer Electrolytes
}

Daniel Sharon, ${ }^{\dagger}$ Peter Bennington, ${ }^{\dagger}$ Michael A. Webb, ${ }^{\|}$Chuting Deng, ${ }^{\dagger}$ Juan J. de Pablo, ${ }^{\dagger}$ Shrayesh N. Patel, $†$ Paul F. Nealey. ${ }^{\dagger}$

${ }^{\dagger}$ Pritzker School of Molecular Engineering, University of Chicago, 5640 S Ellis Ave, Chicago, IL 60637, United States

${ }^{\ddagger}$ Chemical Sciences and Engineering Division and Materials Science Division, Argonne National Laboratory, 9700 South Cass Avenue, Lemont, IL 60439, United States

" Department of Chemical and Biological Engineering, Princeton University, 41 Olden St, Princeton, NJ 08540, United States 


\section{Experimental}

Materials. Block copolymers and homopolymers, SEO block copolymer $\left(M_{n}=9-10 \mathrm{~kg} / \mathrm{mol}\right)$ and PEO homopolymer $\left(M_{n}=20 \mathrm{~kg} / \mathrm{mol}\right)$ were purchased from Polymer Source Inc. and used after vacuum drying at $50^{\circ} \mathrm{C}$ for $24 \mathrm{hr}$. The xPS was received from AZ Electronic Materials. All other chemicals—acetonitrile (99.8\%, anhydrous), toluene, and LiTFSI - were purchased form Sigma Aldrich. The lithium salt was further dried under vacuum at $100^{\circ} \mathrm{C}$. All polymers were stored in an Ar glovebox after the drying processes. The negative photoresist (AZ nLof 2020) and developer (AZ 300 MIF) used for microfabrication of IDEs were purchased from AZ Electronic Materials. Silicon wafers (4" diameter, $500 \mu \mathrm{m}$ thick) with $1 \mu \mathrm{m}$ of thermal $\mathrm{SiO}_{2}$ were purchased from Pure Wafer Inc.

Polymer characterization. Calorimetric glass transition temperatures $\left(T_{\mathrm{g}}\right)$ of neat polymers and polymer electrolytes were determined by DSC (TA Instruments Discovery 2500 DSC). Small-angle X-ray scattering (SAXS) was performed using the SAXSLAB GANESHA instrument at the University of Chicago X-ray Facility. Silicon nitride membranes (100 nm thick, $2.25 \times 2.25 \mathrm{~mm}^{2}$ area) were fabricated in-house to serve as support for the polymer material. Polymer films, with and without salt, were cast from solution onto the $\operatorname{SiN}_{\mathrm{x}}$ membranes and annealed at $165^{\circ} \mathrm{C}$ for 30 minutes. Primary scattering peaks, $q^{*}$, were found by peak fitting using the SAXSGUI MATLAB program developed by SAXSLAB. The BCP domain spacing, $L_{0}$, was taken as $2 \pi / q^{*}$. Tapping mode atomic force microscopy (AFM) was performed using Cypher ES AFM. Vibrational spectroscopy was measured by FTIR (Shimadzu IRTracer-100, attenuated total reflection) and Raman (Horiba LabRAM HR Evo Confocal Raman, 633 nm laser) at room temperature. For these measurement thin films of SEO and PEO LiTFSI were coated on gold coated Si wafer and annealed at $165^{\circ} \mathrm{C}$ inside an argon glovebox.

Electrochemical characterization EIS of the annealed films was characterized using a Gamry Reference 600+. SEO-LiTFSI solutions were prepared inside of an argon glovebox. First, solutions of SEO polymer in acetonitrile and LiTFSI in acetonitrile were prepared and left to stir overnight. Then, these two solutions were mixed at an appropriate ratio to obtain the desired molar ratio of lithium salt to ether oxygen $(\mathrm{r}=[\mathrm{Li}+] /[\mathrm{EO}])$. Thin films were then prepared by spin coating on top of interdigitated electrodes (IDEs). Polymer film height $(h)$ was determined by casting an identical film on a Si wafer and performing ellipsometry (J.A. Woollam alpha-SE ellipsometer). IDEs are microfabricated by photolithography on top of thermal oxide Si wafers. $5 \mathrm{~nm}$ of Ti followed by $45 \mathrm{~nm}$ of Au are deposited in the lithographically defined electrode area, and excess metal is removed by liftoff in warm $n$-methyl pyrrolidone. IDEs are designed with $N=160$ electrodes, $l=1 \mathrm{~mm}$ of electrode overlap, $w=2 \mu \mathrm{m}$ electrode width, and $d=8 \mu \mathrm{m}$ interelectrode distance. IDE surfaces were first homogenized to ensure uniform wetting of the 
substrate and parallel orientation of the lamellae. For asymmetric assembly (PEO wetting of the IDE surface) we used an Ultratech/Cambridge Fiji G2 Plasma-Enhanced ALD to passivate the IDEs with thin layers of $\mathrm{SiO}_{2}(\approx 1 \mathrm{~nm})$. The coated IDEs were mounted on a heating stage and connected to the potentiostat with tungsten probe tips. The temperature of the heating stage was controlled by an Omega controller. The complex impedance spectra for the polymer film was measured by potentiostatic EIS. A $100 \mathrm{mV} \mathrm{AC} \mathrm{potential} \mathrm{is} \mathrm{applied} \mathrm{at} \mathrm{frequencies} \mathrm{ranging} \mathrm{from}$ $1 \mathrm{MHz}$ to $1 \mathrm{~Hz}$. Impedance data was then fit to an appropriate equivalent circuit using the Gamry Echem Analyst software with the simplex algorithm. The film resistance $\left(\mathrm{R}_{\mathrm{f}}\right)$ obtained by this method was then used to determine the ionic conductivity of the polymer electrolyte by the cell constant: $\sigma=\frac{d}{R_{f}(N-1) l h}$. All reported resistance and conductivity values were taken as the average of three samples, with the error bars representing the standard deviation. 


\section{Thermal and structural description of polymer electrolytes}

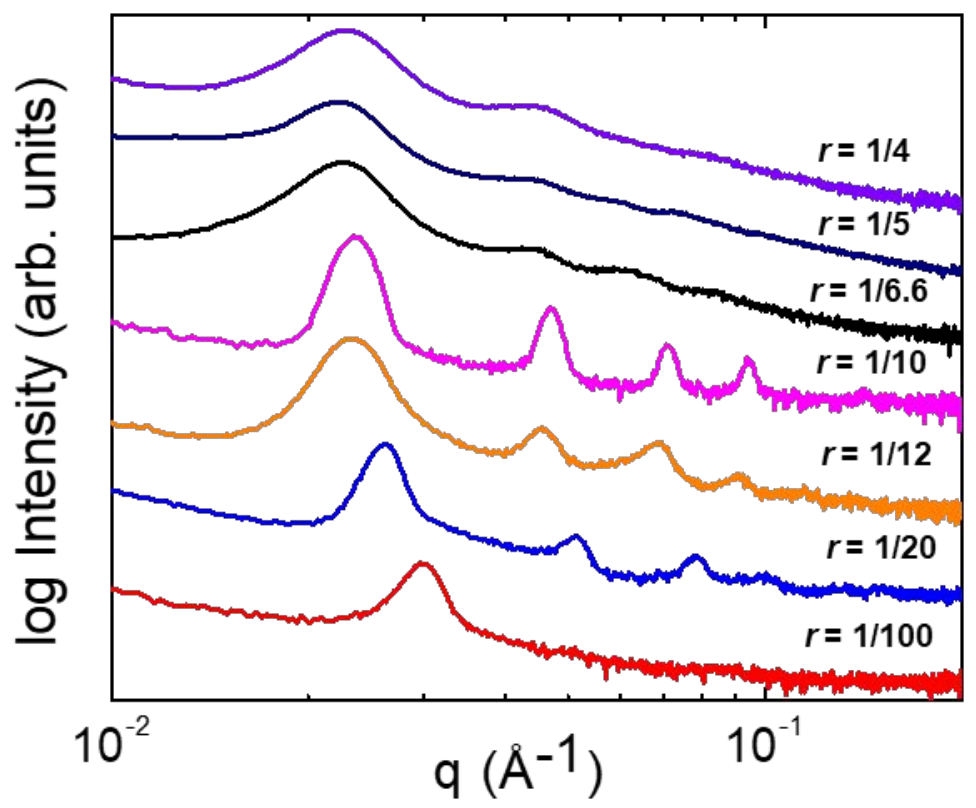

Figure S1. Small angle X-ray (SAXS) diffractions of SEO-LiTFSI 9-10 K electrolytes with different concentration at room temperature after thermal annealing at $165^{\circ} \mathrm{C}$ for 30 minutes

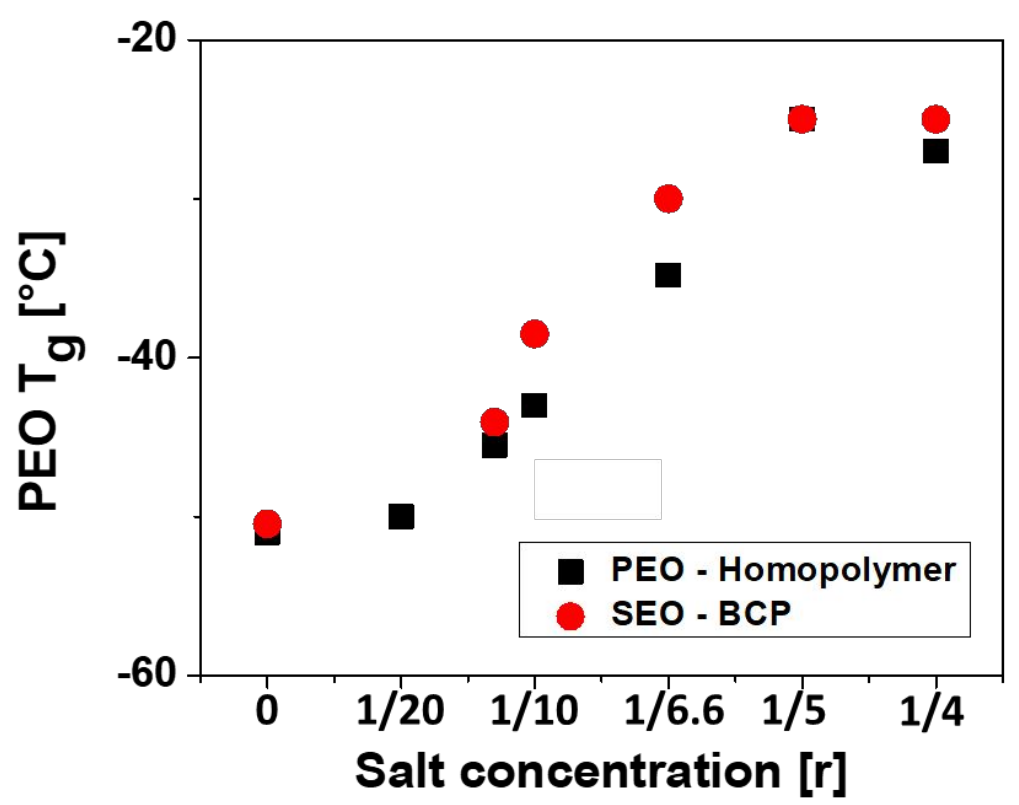

Figure S2. PEO phase glass transition in SEO BCE and PEO homopolymer as function of LiTFSI concentration. 


\section{Volume fraction calculations of conductive phase:}

The conductive phase (PEO + LiTFSI) volume fraction is calculated by the following equation and values ${ }^{1}$ :

$$
\begin{gathered}
\phi_{c}=\frac{\frac{M W_{E O}}{\rho_{E O}}+r \frac{M W_{\text {LiTFSI }}}{\rho_{\text {LiTFSI }}}}{\frac{M W_{E O}}{\rho_{E O}}+r \frac{M W_{\text {LiTFSI }}}{\rho_{\text {LiTFSI }}}+\frac{M W_{P S} M_{E O}}{M W_{P E O} M_{S}} v_{p s}} \\
M W_{E O}=44.05 M W_{P S}=104.05 M W_{\text {LiTFSI }}=104.05 \frac{\mathrm{g}}{\mathrm{mol}} \\
M_{E O}=10,000 M_{P S}=9000 \frac{\mathrm{g}}{\mathrm{mol}} \\
\rho_{E O}=1.128 \rho_{P S}=1.028 \quad \rho_{\text {LiTFSI }}=2.392 \frac{\mathrm{g}}{\mathrm{cm}^{3}}
\end{gathered}
$$

Where $M W_{E O}, M W_{P S}$ and $M W_{L i T F S I}$ are the molecular weights of PEO, PS and LiTFSI, respectively. $M_{E O}$ and $M_{P S}$ are the molar mass of the PEO and PS blocks. $\rho_{E O}, \rho_{P S}$ and $\rho_{\text {LiTFSI }}$ are the density of PEO, PS and LiTFSI, respectively. Table 1 presents the conductive phase volume fraction of the different LiTFSI concentration used in the current study.

\section{Calculation of the effective Flory-Huggins parameter, $\chi_{\text {eff: }}$}

We note, that in the current study we make use of an effective Flory-Huggins parameter, $\chi_{e f f}$, which accounts for the changes in $\chi$ due to the addition of LiTFSI. Additionally, the added volume of the lithium salt will physically swell the system, which gives a higher effective degree of polymerization relative to the same reference volume, $N_{e f f}$ The parameters $\chi_{\text {eff }}$ and $N_{e f f}$ can be determined by using the relationship between the change in the measured $L_{0}$ (from SAXS and AFM) and $(\chi N)_{e f f}$.

The starting point for finding $\chi_{\text {eff }}$ is to determine the value for $\chi$ of the neat PS-PEO BCP. We use $\chi_{S E O}$ that was found in a study by Epps and Bates ${ }^{2}$

$$
\chi_{S E O}=\frac{29.8}{\mathrm{~T}}-22.9 \times 10^{-3}
$$

where $T$ is the absolute temperature (annealing temperature $=165^{\circ} \mathrm{C}$ ). The degree of polymerization, $N$, was calculated by the molecular weights and densities of the PS and PEO blocks which are described above with the respect to a reference volume of $118 \AA^{3}{ }^{3}$. Table S1 summarized the calculated $\chi$ and $N$ for the neat SEO (9-10 K) sample.

Table S1. The properties of SEO BCEs

\begin{tabular}{lllll}
\hline$r([\mathrm{Li}] /[\mathrm{EO}])$ & $L_{0}(\mathrm{~nm})$ & $N_{\text {eff }}$ & $\chi_{\text {eff }}$ & $(\chi N)_{\text {eff }}$ \\
\hline 0 & 18.8 & 248 & 0.045 & 11.2 \\
$1 / 100$ & 21.0 & 252 & 0.082 & 19.6 \\
$1 / 20$ & 24.0 & 267 & 0.145 & 38.4 \\
$1 / 12$ & 26.8 & 281 & 0.232 & 65.2 \\
$1 / 10$ & 26.6 & 286 & 0.202 & 57.9 \\
$1 / 6.6$ & 27.8 & 305 & 0.205 & 62.6 \\
$1 / 5$ & 29.2 & 325 & 0.214 & 69.6 \\
$1 / 4$ & 30.1 & 344 & 0.205 & 70.5
\end{tabular}


In this study, the same SEO BCP was mixed with different amounts of LiTFSI. It is well-documented that LiTFSI preferentially goes into the PEO domain and swells it. Therefore, the increase in $N$ relates only to the PEO phase and can be calculated by ${ }^{4}$ :

$$
N_{\text {eff }}=r \cdot V_{\text {LiTFSI }} \cdot \frac{M_{P E O}}{M W_{P E O}}+N
$$

where $r$ is the molar ration of LiTFSI to ethylene oxide monomers, and $V_{\text {LiTFSI }}$ is the molar volume of the LiTFSI. $V_{\text {LiTFS }}\left(M_{\text {LiTFSI }} / \rho_{\text {LiTfSI }}\right)$ is also normalized by the reference volume of $118 \AA^{3} . N$ is the degree of polymerization of neat (9-10 K) SEO.

By knowing how $N_{\text {eff }}$ varies with salt concentration and using $L_{0}$ data from SAXS measurements, we can use the following equation ${ }^{4}$ to get an effective Flory Huggins parameter:

$$
\chi_{e f f}=\chi \times\left(\frac{L_{0_{L i T F S I}}}{L_{0}}\right)^{6} \times\left(\frac{N}{N_{e f f}}\right)^{4}
$$

where $\chi$ is the Flory-Huggins interaction parameter for the neat polymer which was calculated using Equation S3. Table S1 summarizes the calculated $(\chi N)_{\text {eff }}$ for different salt concentrations of LiTFSI. We note that these values for $\chi_{e f f}$ are in good agreement with previously reported values as a function of salt concentration. ${ }^{5}$

\section{Details of Molecular Dynamics Simulations}

All simulations were performed using the LAMMPS simulation package ${ }^{6}$ with GPU acceleration. ${ }^{7,8}$ Unless otherwise noted, simulations utilized a velocity Verlet integrator with a 1 fs timestep, a Nosé-Hoover thermostat and barostat to control the temperature and pressure, and a 12 Å real-space cutoff for non-bonded interactions; long-range electrostatics are handled using the particle-particle particle-mesh solver. ${ }^{8}$ The force field parameters employed are based on those from Wu and Wick ${ }^{9}$ and include TFSI parameters from Canongia Lopes et al. ${ }^{10}$ Since the majority of the PEO parameters are derived from the TraPPE-UA force field, ${ }^{11}$ parameters for polystyrene atoms were also taken from the TraPPE-UA force field ${ }^{12-14}$ for consistency. Example LAMMPS data files that also exemplify all the force field parameters used are provided for each concentration studied as supplementary files. The procedures used for system preparation and simulation is described in subsequent sections.

Initial chain generation. To begin, 100 independent replicas of SEO chains were produced in a united-atom representation using an in-house code. The molecular weight of each polymer chain was chosen to be commensurate with experimental samples, such that each chain features 238 ethylene oxide units (approximately $10472 \mathrm{Da}$ ) followed by 90 sytrene units (approximately $9369 \mathrm{Da}$ ); the ends of each polymer chain were terminated by methyl groups. The polystyrene portion of the polymer chain is atactic, with either stereochemical styrene unit stochastically chosen at each polymerization step with equal probability. The polymer chains were initially generated in mostly extended but randomized configurations wherein a vector in the direction of the backbone of each monomer undergoes random rotations in the $\mathrm{x}, \mathrm{y}$, and $\mathrm{z}$ direction (in random order) by a random angle between $-70^{\circ}$ and $+70^{\circ}$ during the computational polymerizaion. Following their initial generation, each polymer chain was relaxed and further randomized by performing $10 \mathrm{~ns}$ of Langevin dynamics (damping parameter of 1000 fs) in an infinite medium with a dielectric constant of 4; the temperature, which is controlled by the random implicit solvent forces, was ramped linearly from $800 \mathrm{~K}$ to $400 \mathrm{~K}$ during this initial equilibration phase.

Initial block copolymer lamellar assembly. The 100 previously generated SEO chains are combined into a single simulation cell to set up an initial block copolymer morphology. To facilitate the assembly of a lamellar morphology, two interfacial planes perpendicular to the $z$-axis are defined within the simulation cell at $z= \pm 5 \mathrm{~nm}$, and a 10x10 square lattice is generated for each plane on the domain of $x \in[0,12.5]$ and $y \in[0,12.5] \mathrm{nm}$. Afterwards, each chain is placed in the simulation cell according to the following procedure: (i) a random polymer chain is selected, and its configuration is rotated such that the vector adjoining the first and last atom in the chain is aligned with the $z$-axis; (ii) one of the two interfacial planes is randomly chosen with equal probability; (iii) an unoccupied lattice point is chosen at random, (iv) the polymer is translated such that the first carbon atom of the EO unit nearest to the PS block (also referred to as the "anchor atom") is placed at the position of the lattice point; (v) and the polymer is translated in the $z$-direction according to a probability distribution that approximately yields an interface with the functional form 


$$
\phi_{\mathrm{EO}}(z)=\frac{1}{2}\left(1 \pm \tanh \frac{2(z \mp 5 \mathrm{~nm})}{\Delta}\right)
$$

where $\phi_{\mathrm{EO}}(z)$ is the fraction of EO atoms amongst all atoms at a particular value of $z, z^{*}=5 \mathrm{~nm}$, and $\Delta=2 \mathrm{~nm}$; the use of the top signs or bottom signs depends on which of the two planes is chosen in (ii). The output of this procedure is an unequilibrated configuration of SEO chains that are largely segregated into PEO-rich and PS-rich domains with some finite interfacial width.

Neat block copolymer annealing and equilibration. The simulation cell for the previously generated configuration was initially set as $\mathrm{x} \in[0,12.5], \mathrm{y} \in[0,12.5]$, and $\mathrm{z} \in[-12.5,12.5]$. To preserve some interfacial width provided by the initial configuration, harmonic restraints of the from $K\left(z-z_{o}\right)^{2}$ were applied to all anchor atoms, where $z_{o}$ is the initial z-coordinate of the anchor atom; the spring constant for these restraints, $K$, was set to be 50 $\mathrm{kcal} /(\mathrm{mol} \AA$ ) $)$. Any significant overlaps among particles were then removed by running 1000 steps of NVE dynamics, during which the maximum allowable displacement for any given particle per timestep was limited to $0.05 \AA$. Next, the system was annealed at constant volume from $300 \mathrm{~K}$ to $600 \mathrm{~K}$ over $5 \mathrm{~ns}$ (timestep set to $0.5 \mathrm{fs}$ ), and the temperature was then maintained at $600 \mathrm{~K}$ for an additional $5 \mathrm{~ns}$ using a Berendsen thermostat with a 100 -fs relaxation time. At this point, the harmonic restraints were removed from the anchor atoms, and two 10-ns cooling cycles were performed under NPT conditions (pressure target of $1 \mathrm{~atm}$ ). Specifically, the system was cooled from $600 \mathrm{~K}$ to $400 \mathrm{~K}$ over $10 \mathrm{~ns}$, instantaneously heated back to $600 \mathrm{~K}$, and then cooled back to $400 \mathrm{~K}$ over $10 \mathrm{~ns}$. The system was then equilibrated under NPT conditions for $40 \mathrm{~ns}$ at $393 \mathrm{~K}$ with a $2.0 \mathrm{fs}$ timestep. The average density over the last $12 \mathrm{~ns}$ of this equilibration is $1.048 \mathrm{~g} / \mathrm{cm}^{3}$, and the final simulation cell dimensions were $12.88 \mathrm{~nm} \times$ $12.88 \mathrm{~nm} \times 12.88 \mathrm{~nm} 19.13 \mathrm{~nm}$.

Salt insertion and additional equilibration. To obtain systems at various salt concentrations, ions are randomly inserted into defined domains of partially equilibrated systems. In particular, to span the range of dilute to high-salt conditions, configurations were prepared for $r \approx 0.01,0.05,0.085,0.15$, and 0.25 through the addition of 238, 1190, 2023,3570 , and 5950 LiTFSI pairs to the neat polymer system. To facilitate equilibration, these systems were generated in series, such that the initial seed configuration for $r \approx 0.01$ was taken from the neat polymer, the initial configuration for $r \approx 0.05$ was taken from $r \approx 0.01$, and so on; each of these seed configurations was taken as the configuration after $20 \mathrm{~ns}$ of NPT simulation at $393 \mathrm{~K}$ and $1 \mathrm{~atm}$. After obtaining a seed configuration, the center-ofmass coordinates for the requisite number of anions and cations to be added and achieve the desired concentration were selected from a uniform random distribution on the intervals $[0,12.88] \mathrm{nm},[0,12.88] \mathrm{nm}$, and a variable domain for the $x, y$, and $z$ dimensions, respectively; the domain of the $z$-coordinate placement is taken to be the inner $40 \%$ of the simulation cell, where the polymer composition is nearly $100 \%$ PEO. Since the ions were distributed randomly and uniformly over the prescribed intervals, inhomogeneities in ion distribution observed subsequently can be attributed to the system evolving to more preferred, equilibrium configurations. The random ion placement results in highly overlapping configurations, which were removed by running 1000 steps of NVE dynamics with maximum allowable displacement for any given particle per timestep limited to $0.05 \AA$. Then, these systems were equilibrated under NPT conditions for 40 ns (with seed configurations taken at 20 ns, if necessary, as previously discussed).

Production run and analysis. Following the equilibration described above, simulations for $r \approx 0.01,0.05$, and 0.085 systems were run for $72 \mathrm{~ns}$ and those for $r \approx 0.15$ and 0.25 were run for $54 \mathrm{~ns}$. The final $50 \mathrm{~ns}$, for each salt concentration, was used for analysis. Figure 5 of the main text presents simulation results that resolve the distribution of species along the $z$ axis of the simulation cell. These compositional profiles were determined by construction of particle number histograms with a bin width of $1 \AA$; configurations were sampled for analysis every $0.6 \mathrm{~ns}$.

\section{References}

1. Timachova, K.; Villaluenga, I.; Cirrincione, L.; Gobet, M.; Bhattacharya, R.; Jiang, X.; Newman, J.; Madsen, L. A.; Greenbaum, S. G.; Balsara, N. P. Anisotropic Ion Diffusion and Electrochemically Driven Transport in Nanostructured Block Copolymer Electrolytes. J. Phys. Chem. B 2018, 122 (4), 1537-1544.

2. Epps, T. H.; Bailey, T. S.; Waletzko, R.; Bates, F. S. Phase Behavior and Block Sequence Effects in Lithium Perchlorate-Doped Poly(Isoprene-b-Styrene-b-Ethylene Oxide) and Poly(Styrene-b-Isoprene-b-Ethylene Oxide) Triblock Copolymers. Macromolecules 2003, 36 (8), 2873-2881.

3. Cochran, E. W.; Morse, D. C.; Bates, F. S. Design of ABC Triblock Copolymers near the ODT with the Random Phase Approximation. Macromolecules 2003, 36 (3), 782-792. 
4.Chen, X.; Zhou, C.; Chen, S. J.; Craig, G. S. W.; Rincon-Delgadillo, P.; Dazai, T.; Miyagi, K.; Maehashi, T.; Yamazaki, A.; Gronheid, R.; Stoykovich, M. P.; Nealey, P. F. Ionic Liquids as Additives to Polystyrene- BlockPoly(Methyl Methacrylate) Enabling Directed Self-Assembly of Patterns with Sub-10 Nm Features. ACS Appl. Mater. Interfaces 2018, 10 (19), 16747-16759.

5. Loo, W. S.; Galluzzo, M. D.; Li, X.; Maslyn, J. A.; Oh, H. J.; Mongcopa, K. I.; Zhu, C.; Wang, A. A.; Wang, X.; Garetz, B. A.; Balsara, N. P. Phase Behavior of Mixtures of Block Copolymers and a Lithium Salt. J. Phys. Chem. B 2018, 122 (33), 8065-8074.

6.Plimpton, S. Fast Parallel Algorithms for Short-Range Molecular Dynamics J. Comput. Phys. 1995, 117, 119 DOI: 10.1006/jcph.1995.1039

7. Brown, W. M.; Wang, P.; Plimpton, S. J.; Tharrington, A. N. Implementing Molecular Dynamics on Hybrid High Performance Computers - Short Range Forces Comput. Phys. Commun. 2011, 182, 898- 911.

8. Brown, W. M.; Kohlmeyer, A.; Plimpton, S. J.; Tharrington, A. N. Implementing Molecular Dynamics on Hybrid High Performance Computers - Particle-Particle Particle-Mesh Comput. Phys. Commun. 2012, 183, 449- 459.

9. Wu, H.; Wick, C. D. Computational Investigation on the Role of Plasticizers on Ion Conductivity in Poly(ethylene oxide) LiTFSI Electrolytes Macromolecules 2010, 43, 3502- 3510

10. Canongia Lopes, J. N.; Pádua, A. A. H. Molecular Force Field for Ionic Liquids Composed of Triflate or Bistriflylimide Anions. J. Phys. Chem. B 2004, 108, 43, 16893-16898.

11. Stubbs, J. M.; Potoff, J. J.; Siepmann, J. I. Transferable Potentials for Phase Equilibria. 6. United-Atom Description for Ethers, Glycols, Ketones, and Aldehydes J. Phys. Chem. B 2004, 108, 17596- 17605

12. Martin, M.G.; Siepmann, J.I. Transferable potentials for phase equilibria. 1. United-atom description of nalkanes. J. Phys. Chem. B 1998, 102, 2569-2577.

13. Martin, M.G.; Siepmann, J.I.Novel configurational-bias Monte Carlo method for branched molecules. Transferable potentials for phase equilibria. 2. United-atom description of branched alkanes. J. Phys. Chem. B 1999 $103,4508-4517$.

14. Wick, C.G; Martin, M.G.; Siepmann, J.I. Transferable potentials for phase equilibria. 4. United-atom description of linear and branched alkenes and of alkylbenzenes. J. Phys. Chem. B 2000, 104, 8008-8016. 